\title{
Selective translational usage of TSS and core promoters revealed by translatome sequencing
}

Hua $\mathrm{Li}^{i^{*+}} \mathbb{D}$, Ling Bai ${ }^{1 * \dagger}$, Hongmei $\mathrm{Li}^{2}$, Xinhui $\mathrm{Li}^{1}$, Yani Kang ${ }^{1}$, Ningbo Zhang ${ }^{3}$, Jielin Sun ${ }^{3}$ and Zhifeng Shao ${ }^{1}$

\begin{abstract}
Background: In mammals, fine-tuned regulation of gene expression leads to transcription initiation from diverse transcription start sites (TSSs) and multiple core promoters. Although polysome association is a critical step in translation, whether polysome selectively uses TSSs and core promoters and how this could impact translation remains elusive.
\end{abstract}

Results: In this study, we used CAGE followed by deep sequencing to globally profile the transcript 5' isoforms in the translatome and transcriptome of human HEK293 cells at single-nucleotide resolution. By comparing the two profiles, we identified the $5^{\prime}$ isoforms preferentially used in translatome and revealed a widespread selective usage of TSSs (32.0\%) and core promoters (48.7\%) by polysome. We discovered the transcription initiation patterns and the sequence characteristics that were highly correlated with polysome selection. We further identified 5804 genes significantly enriched or depleted in translatome and showed that polysome selection was an important contributing factor to the abundance of related gene products. Moreover, after comparison with public transcriptome CAGE data from 180 human tissues and primary cells, we raised a question on whether it is a widely adopted mechanism to regulate translation efficiency by changing the transcription initiation sites on the transcription level in cells of different conditions.

Conclusions: Using HEK293 cells as a model, we delineated an indirect selection toward TSSs and core promoters by the translation machinery. Our findings lend additional evidence for a much closer coordination between transcription and translation, warranting future translatome studies in more cell types and conditions to develop a more intricate regulatory model for gene expression.

Keywords: CAGE, Translatome sequencing, TSS profiling, Core promoter, Polysome selection

\section{Background}

The flow of genetic information is tightly controlled at multiple levels to maintain proper phenotypes and achieve cellular fitness. The regulation of transcription, the first step in gene expression, has been extensively studied and its complexity has been elaborated mostly owing to highly efficient next-generation sequencing techniques [1-3]. Besides transcriptional regulation, it is now more evident that translational regulation on mRNA also has substantial

\footnotetext{
* Correspondence: kaikaixinxin@sjtu.edu.cn; Ibai@sjtu.edu.cn

${ }^{+} H u a$ Li and Ling Bai contributed equally to this work.

${ }^{1}$ State Key laboratory for Oncogenes and Bio-ID Center, School of Biomedical Engineering, Shanghai Jiao Tong University, 800 Dongchuan Road, Shanghai 200240, China

Full list of author information is available at the end of the article
}

control over gene expression by modulating mRNA translation, stability and localization $[4,5]$ Translational regulatory factors constitute a highly complex network to control protein product and output, thus playing critical roles in cellular metabolisms and tumorigenesis [6-9].

The noncoding part of mRNA, including the $5^{\prime}$ UTR (with the $5^{\prime}$ cap), the $3^{\prime}$ UTR and the poly(A) tail, is responsible for most translational regulation on gene expression. The 5' UTR is of special importance to translational initiation where protein synthesis is principally regulated: during the initiation step in eukaryotes, eukaryotic initiation factors recruit the small ribosomal subunit (40S) to form a pre-initiation complex that scans the $5^{\prime}$ UTR to locate the start codon, after which the initiation factors are released and the large ribosomal subunit (60S) is recruited

(c) The Author(s). 2019 Open Access This article is distributed under the terms of the Creative Commons Attribution 4.0 International License (http://creativecommons.org/licenses/by/4.0/), which permits unrestricted use, distribution, and 
to form the elongation-competent $80 \mathrm{~S}$ ribosome [10]. Several features in the $5^{\prime}$ UTR, such as the $5^{\prime}$ cap, secondary structure and length, are known to affect translation [11, 12]. Other important regulatory features, such as upstream AUGs (uAUGs), have also been studied in recent genome-wide studies $[4,13,14]$.

Cap Analysis of Gene Expression (CAGE) is a powerful method widely used to profile the $5^{\prime}$ ends in organisms like human, fly and yeast $[1-3,15-18]$. Accumulated CAGE data have clearly shown that a single gene can have highly heterogeneous $5^{\prime}$ ends (i.e., $5^{\prime}$ isoforms) in total RNA (i.e., transcriptome). This heterogeneity is one manifestation of the complex transcriptional regulation in eukaryotes: the transcription machinery employs diverse transcription start sites (TSSs) and multiple core promoters to precisely and dynamically regulate gene transcription [19-21]. Besides, selective usage of TSSs and core promoters in transcription could have great impact on translation, thus altering the abundance of protein products or even changing the related biological functions [11, 19-21]. By contrast, systematic investigation on $5^{\prime}$ ends of polysome-associated RNAs (i.e., translatome), which is more closely related to translation process and protein products, has only been performed in a very limited scale $[4,11,22]$. Given the importance of the $5^{\prime}$ UTR, the lack of information on the difference between translatome and transcriptome will limit our ability to decipher the sophisticated regulatory mechanisms in translation.

In this study, we employed CAGE followed by deep sequencing to globally profile the transcript $5^{\prime}$ isoforms in the translatome of human HEK293 cells at single-nucleotide resolution. This allowed us to precisely annotate the $5^{\prime}$ ends, portray the $5^{\prime}$ end distributions, define the $5^{\prime}$ UTR and identify core promoters used by polysome. By comparing them with the counterparts from HEK293's transcriptome, we revealed selective usage of the TSS-derived $5^{\prime}$ ends by polysome, thus delineating an indirect selection toward TSSs and core promoters by the translation machinery. In addition, quantitative measurement of transcript abundance with CAGE allowed us to investigate the transcript enrichment after polysome selection, enabling the identification of highly enriched or depleted gene products in translatome. All these differences between transcriptome and translatome highlight the important roles of polysome in regulating gene expression, the interplay between transcription and translation and the necessity of developing a more intricate model to explain the underlining mechanisms.

\section{Results and discussion}

\section{The landscape of $5^{\prime}$ transcript ends in translatome and} transcriptome

A flowchart of our work before data analysis is shown in Fig. 1a. CAGE tags from deep sequencing were processed with fqtrim to remove low-quality ones, generating approximately 18 million and 14 million tags respectively for the translatome and transcriptome of HEK293 [23]. These tags were then mapped to the human genome (assembly GRCh37) using bowtie with two mismatches allowed [24]. Tags mapped to rRNA were less than $17.9 \%$ for translatome and $7.5 \%$ for transcriptome, indicating high quality of the two CAGE libraries $[3,16]$. In total, $6,973,108$ tags $(37.9 \%)$ for translatome and $6,791,846$ tags $(49.3 \%)$ for transcriptome were uniquely mapped and used for downstream analysis. The vast majority of CAGE tags were located within $100 \mathrm{nt}$ flanking the $5^{\prime}$ ends of known transcripts in both translatome (79.4\%) and transcriptome (72.0\%) (Additional file 1: Figure S1), which was consistent with previous studies $[16,22,25]$. All tags were mapped to 804,594 and $1,315,195$ unique genomic positions, with top 100,000 positions $(<0.01 \%$ of the human genome) representing 83.0 and $70.9 \%$ of all tags in translatome and transcriptome, respectively. These results showed a significant aggregation of CAGE tags and an excellent agreement between our data and existing annotations. They also showed that, the number of unique $5^{\prime}$ ends in translatome is much less than in transcriptome, which was a highly expected result.

We merged overlapped CAGE tags (at least 4 tags) into tag clusters (TC) and each TC represents a putative core promoter (see Methods) [3, 16]. In total, we identified 29,908 and 37,530 TCs, consisting of 97.0 and $90.9 \%$ CAGE tags in translatome and transcriptome, respectively. Using the $5^{\prime}$ end of the most redundant tag in a TC to represent the position of the $\mathrm{TC}$, we calculated the distribution of TCs across four annotated genomic features of all protein-coding genes (Fig. 1b; unless otherwise specified, we used "genes" hereafter to refer to protein-coding genes only). In translatome, we observed a much higher proportion (59.0\%) of TCs located within $5^{\prime}$ UTRs than in transcriptome (49.3\%; $p$-value $<0.001$ by proportion test), suggesting that transcripts with canonical ORFs were more likely to be translated. We identified a considerable fraction of TCs from intron and the coding sequence (CDS) in transcriptome (similar to the findings in human $[16,22$, 25]; Fig. 1b), most of which were also present in translatome. Although it is unclear what proportion of these TCs would be further translated, recent studies have already highlighted the biological significance of the resulting truncated peptides [19, 20, 26, 27].

\section{Selective usage of TSS by Polysome}

In human, transcription usually initiates from multiple positions (i.e., TSSs) within core promoters, resulting in diverse distribution patterns of $5^{\prime}$ transcript ends in transcriptome $[3,16,25,28]$. As expected, using a method proposed in previous studies [3, 16], we were able to classify the TCs of transcriptome into 4 shape 


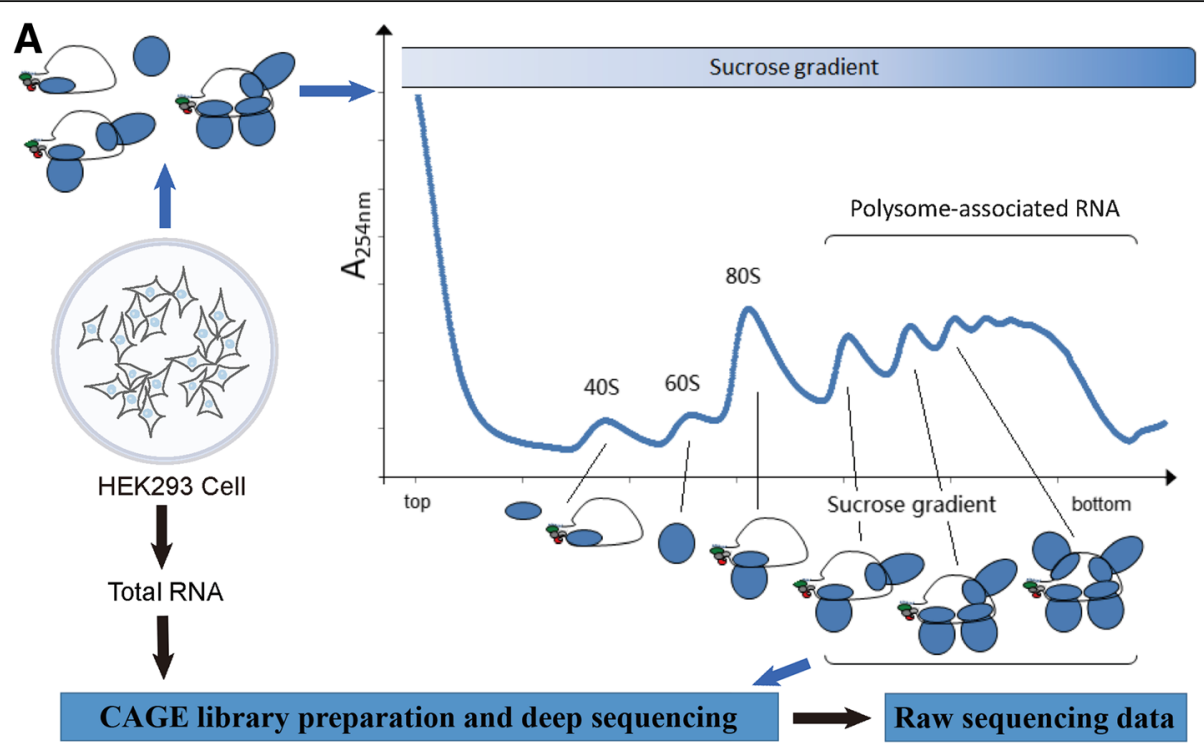

B

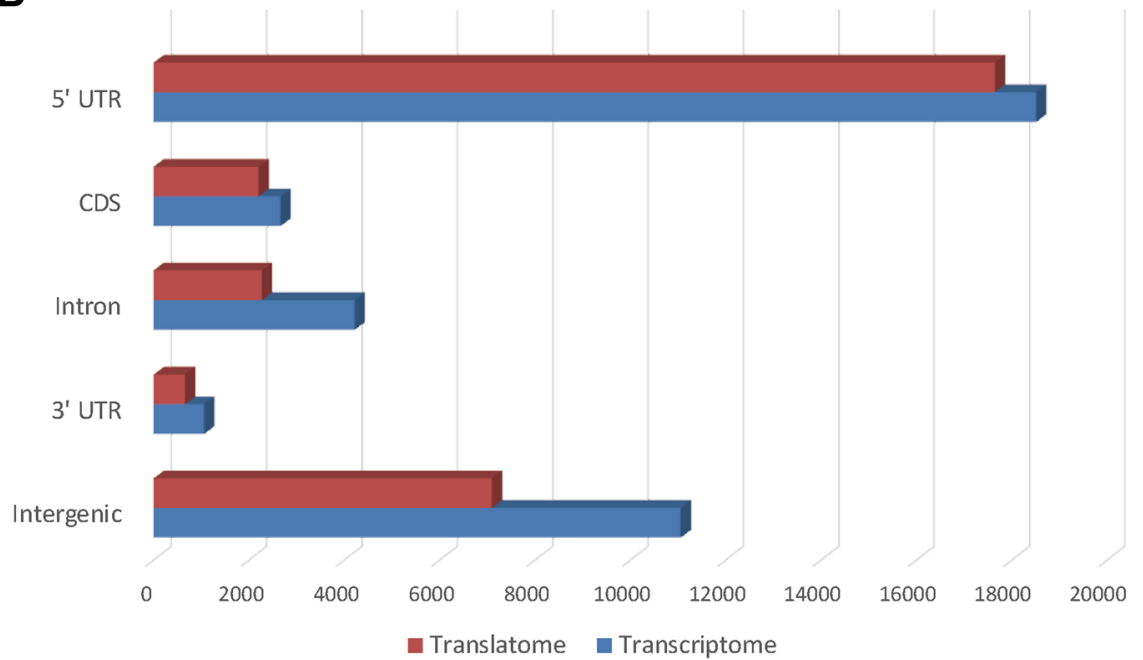

Fig. 1 A flowchart of our work and the distribution of TCs. a A flowchart that briefly describes the steps to obtain the raw sequencing data. $\mathbf{b}$ Distribution of TCs across different genomic features in HEK293 cell line. Four genomic features (5' UTR, 3' UTR, CDS and intron) of all proteincoding genes were based on human GRCh37 annotations from Ensembl. The rest of the genome were considered "intergenic" (see Methods)

classes based on the $5^{\prime}$ end distributions (Additional file 2: Figure S2). The 4 classes are (i) single dominant peak (SP), (ii) broad with a single dominant peak (DP), (iii) broad with bi- or multi- peaks (MP), and (iv) generally broad peaks (BP), the proportions of which are comparable to previous studies [16, 25]. We hereafter only analyzed TCs located in the annotated 5' UTRs (including the upstream $100 \mathrm{nt}$ ) since these TCs and their internal $5^{\prime}$ ends corresponded to core promoters and TSSs, respectively $[3,16]$. Very importantly, although we were also able to classify the TCs of translatome into these 4 classes, the $5^{\prime}$ end (i.e., TSS) distributions for a large proportion of TCs changed from their counterparts in transcriptome. This distribution disparity was assessed using Kolmogorov-Smirnov test (KS test) for TCs with at least 100 tags in both translatome and transcriptome. To our surprise, as many as 1781 TCs $(32.0 \%)$ underwent $5^{\prime}$ end distribution change ( $p$-value $<0.001$ by KS test; Fig. 2a), of which 775 (43.5\%) had different shape classes between translatome and transcriptome (Additional file 3: Table S1). These results demonstrate that preferential usage of TSSs by polysome is a widespread phenomenon in HEK293 cell line.

The position of the highest density in the $5^{\prime}$ end distribution, which corresponds to the most frequently used TSS (defined as representative TSS) within a core promoter, was used in previous work to define the $5^{\prime}$ UTR [3]. In this study, representative TSSs and the $5^{\prime}$ UTR 

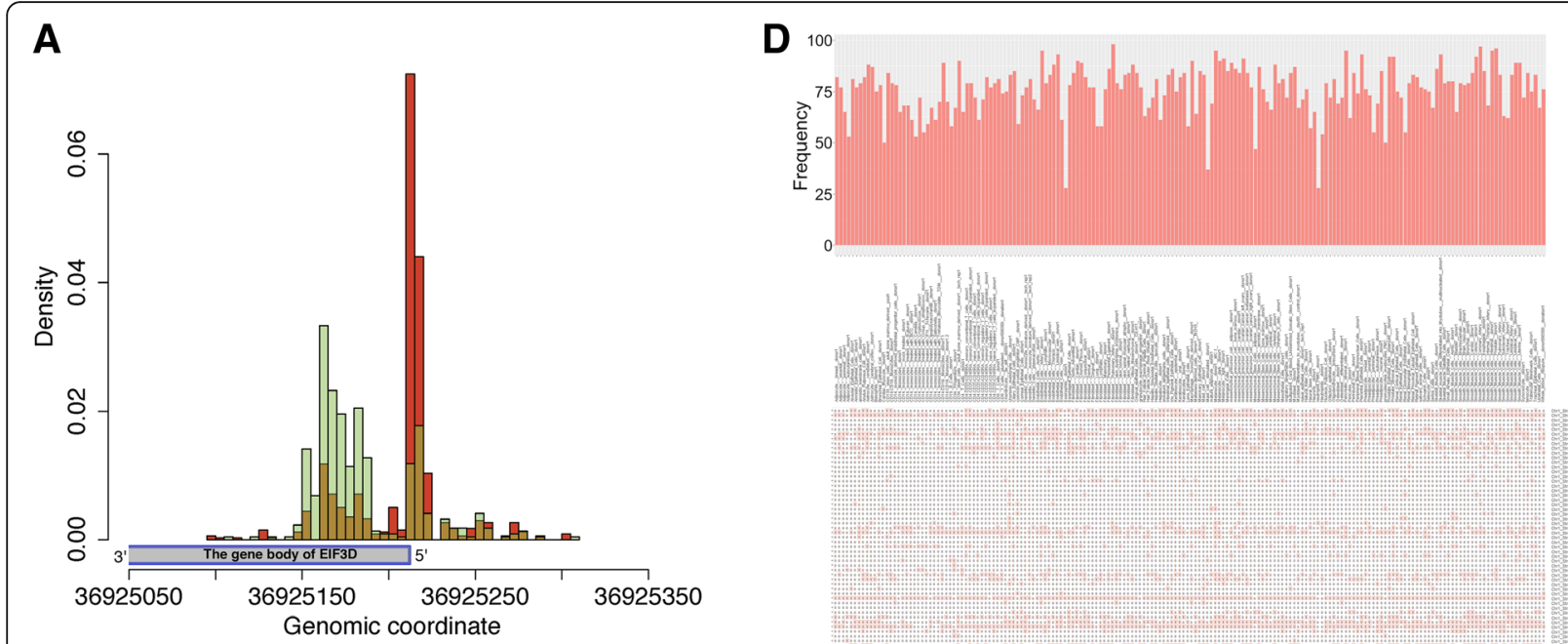

\section{B}

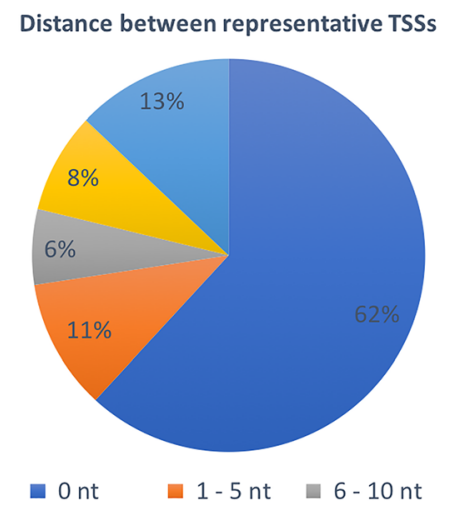

घ $11-20 \mathrm{nt} \square>20 \mathrm{nt}$

C

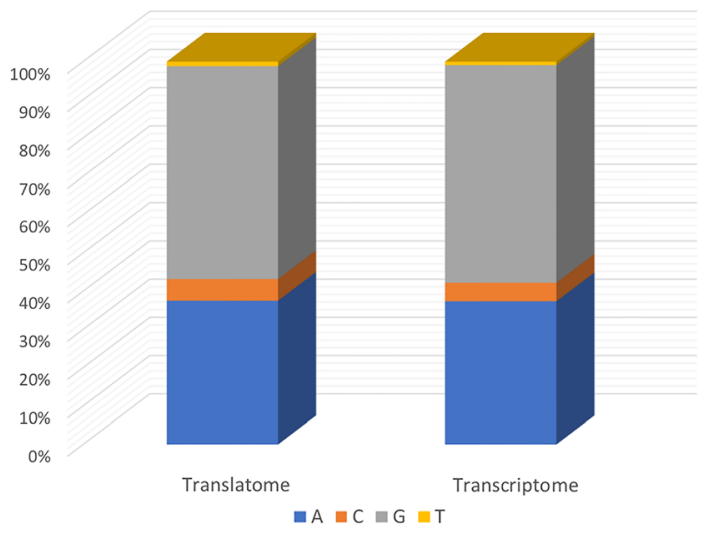

Fig. 2 (See legend on next page.) 
(See figure on previous page.)

Fig. 2 Comparison of TSSs between translatome and transcriptome. a A typical example of TSS distribution disparity. The two TCS are located in at the core promoter region of the gene "EIF3D". The red color stands for the $5^{\prime}$ end distribution in translatome and the green stands for the distribution in transcriptome (the yellow is the common part of the two distributions). $\mathbf{b}$ The distance between representative TSSs of translatome and transcriptome. The distance is calculated with the genomic coordinates of representative TSSs on the human genome. c Comparison of the nucleotide frequency at representative TSSs in translatome and transcriptome. d Usage landscape of HEK293-derived representative TSSs in 180 human samples. All 3248 representative TSSs are from HEK293 translatome and only those from chromosome 1 are displayed here for better visualization. In the heatmap, "1" (marked with red) means the representative TSS on the right side is also used as representative TSS in the sample on the top. The histogram on top of sample names shows the number of representative TSSs used by each sample. The name of each representative TSS contains the information of chromosome, strand and genomic position. For the full list of all 3248 representative TSSs, please refer to Additional file 5: Table S3

were defined in the same way, and length difference of the $5^{\prime}$ UTR was calculated between translatome and transcriptome (Additional file 4: Table S2). Among the 8513 TCs with at least 10 tags mapped to the representative TSSs, 38.2\% (3248) had different representative TSSs between translatome and transcriptome (Fig. 2b); by contrast, only $20.3 \%$ of the 8513 TCs (much lower than $38.2 \%$; $p$-value $<0.001$ by proportion test) used different nucleotides at the representative TSSs, showing conservations that were consistent with previous findings that the identity of the first nucleotide had a strong influence on the translation of the corresponding transcript [20]. In addition, in transcriptome, the nucleotide frequencies at the representative TSSs were similar to those in previous studies [16, 25]; in translatome, the frequencies were largely the same, except that the frequency of "C" increased appreciably (1.2-fold change, p-value $<0.05$ by proportion test; Fig. $2 \mathrm{c}$ ).

We downloaded deep sequencing-derived CAGE data from the FANTOM5 project (phase 1.3 and 2.0), which included the TCs and representative TSSs in transcriptome for 180 human tissues and primary cells [29]. We compared the representative TSSs between the HEK293 translatome and the 180-sample CAGE data. We found that, almost $90 \%$ of the aforementioned 3248 representative TSSs could be found in at least 1 of the 180 transcriptome CAGE data as representative TSSs (Fig. 2d; Additional file 5: Table S3). Since the representative TSSs in translatome shows the most preferred $5^{\prime}$ transcript ends by polysome, exactly matched representative TSSs between transcriptome and translatome should contribute to better efficiency for polysome association, thus probably enhancing translational efficiency. Therefore, based on these observations, we raised a question worth further investigations: could it be a widely adopted mechanism to regulate translation by employing different representative TSSs at the transcription level in different cell types?

\section{Selective usage of core promoters by Polysome}

In eukaryotes from yeast to human, a single gene could use multiple core promoters in transcription as a result of complex gene expression regulation [3, 16, 25]. Here in HEK293 cell line, $37.2 \%$ of the expressed genes used at least 2 core promoters to initiate their transcription
(Fig. 3a); by contrast, the percentage of genes still using $\geq 2$ core promoters went down significantly in translatome $(25.3 \%, p$-value $<0.001$ by proportion test). Although the majority of core promoter-derived TCs were also associated with polysome (Fig. 3b), their abundance (measure by Reads Per Million - RPM) could be changed significantly on polysome (see the next paragraph). An unneglectable fraction (17.5\%) of core promoter-derived TCs were not present in translatome, and their average RPM were much lower than that of the others in transcriptome ( $p$-value $<0.001$ by Wilcoxon test; Fig. 3c). These results suggest that preferential usage of core promoters is a common phenomenon for polysome in HEK293 cell line.

We found a surprisingly high proportion $(48.6 \%)$ of TCs with significantly changed RPM $\left(\left|\log _{2} \mathrm{FC}\right| \geq 1\right.$ and $p$-value $<0.05$ by the $\mathrm{R}$ package of DEGseq, where FC (i.e., fold change) is defined as $\mathrm{RPM}_{\text {translatome }} / \mathrm{RPM}_{\text {tran- }}$ scriptome for each TC) between translatome and transcriptome [30]. Among these TCs (Additional file 6: Table S4), only 2488 (25.8\%) showed enrichment in translatome, corroborating the preferential usage of certain core promoters in translation (see Additional file 7). Moreover, for translatome-enrich TCs, we discovered a significant correlation between transcription initiation pattern and TC enrichment level (Fig. 3d): when the TC fold change went higher, the percentage of TCs in SP class generally went much higher (Pearson correlation $R=0.94, p$-value $<0.001)$ while the percentage of $\mathrm{BP}$ class generally became much lower $(R=-0.95, p$-value $<$ $0.001)$. Since the shape class of the same TC may change between different types of cells [25, 28, 29], it could be a potential strategy to regulate translation by controlling the transcription initiation pattern.

We compared the immediate downstream sequences $(100 \mathrm{nt})$ of representative TSSs between top-enriched 200 TCs, top-depleted 200 TCs and randomly picked 200 TCs with FC between 0.95 and 1.05. We first used WebLogo to examine the sequence features and found that the top-enriched TCs had the least GC content while the top-depleted ones had the most (Fig. 3e) [31]. By counting the total number of AUG (the start codon) in the $100 \mathrm{nt}$ sequence for each group, we found that the top-enriched TCs had significantly more AUG (151) 
A

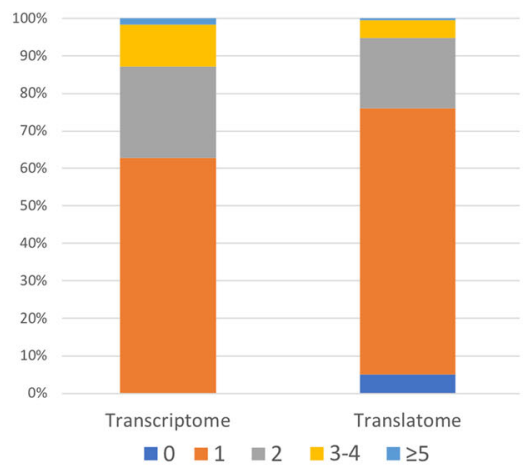

C

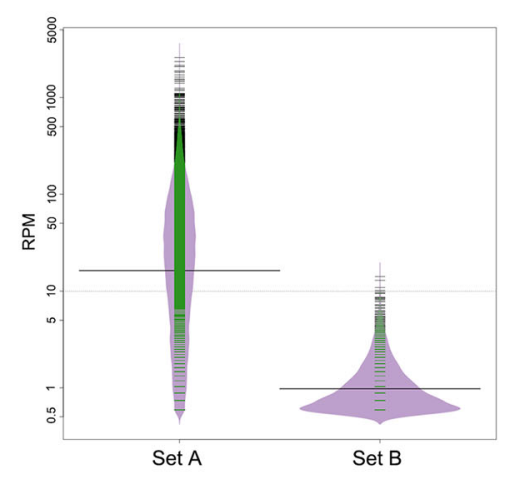

E

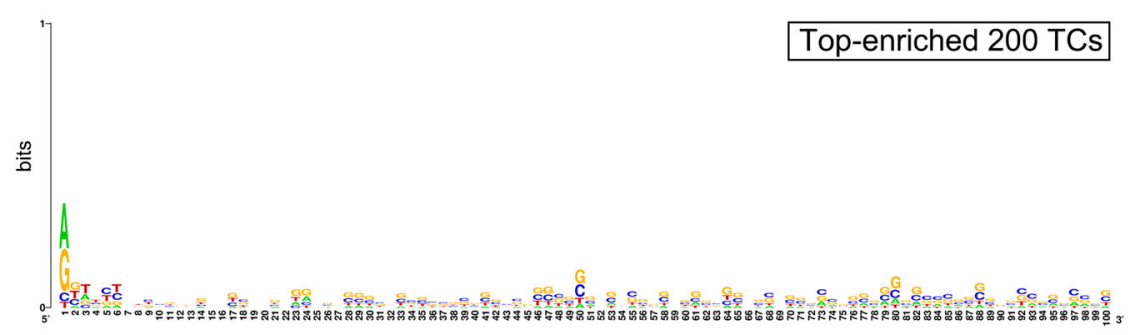

B

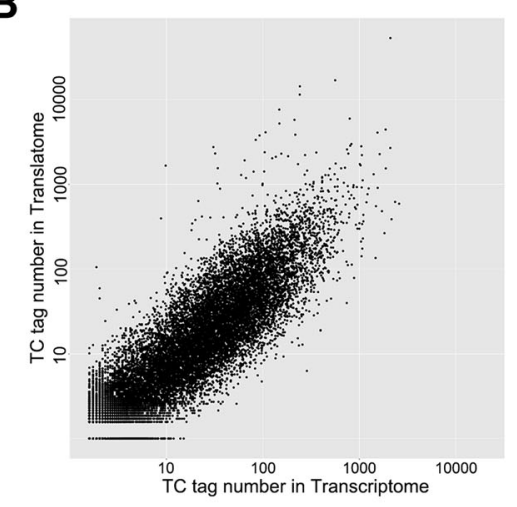

$\mathbf{D}_{900}$

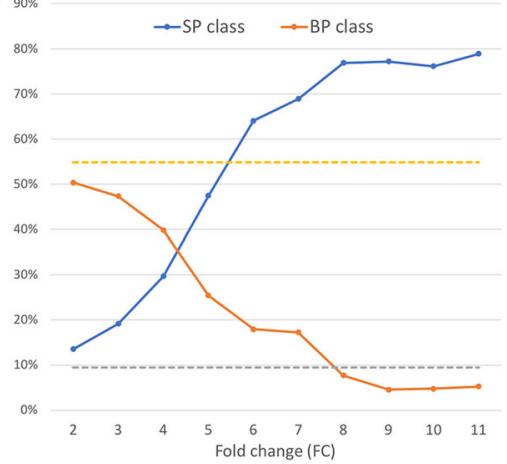

Top-enriched 200 TCs

Randomly picked $200 \mathrm{TCs}$

$\frac{2}{\frac{2}{0}}$
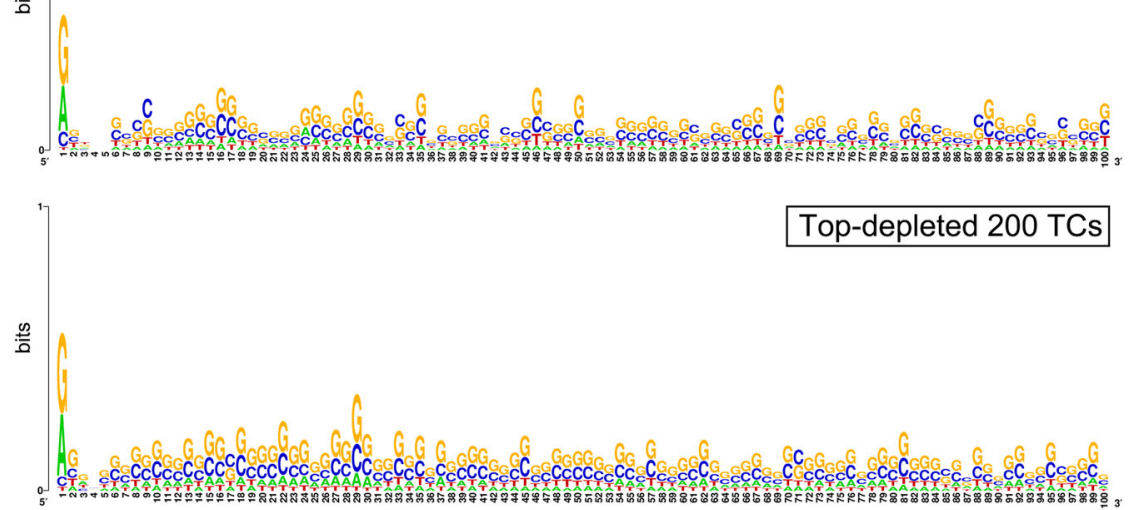

Fig. 3 (See legend on next page.) 
(See figure on previous page.)

Fig. 3 Comparison of core promoter usage in translatome and transcriptome. a The percentage of genes using specific number of core promoters. $\mathbf{b}$ Scatterplot of the TC tag numbers in translatome and transcriptome. As the two axes are in logarithmic scale, the number of tags in all TCs has been increased by 1 to avoid 0. $\mathbf{c}$ Beanplot of the TC abundance measured by RPM. Among the TCs in transcriptome, those present in translatome are grouped into "Set A" while those not present are grouped into "Set B". In this plot, the short green lines mark the observations of RPM, while the purple area shows the frequency of the observed RPM. The two long solid black lines stand for the average of each set and the long dotted black line stands for the overall average of two sets. $\mathbf{d}$ Correlation between TC fold change and TC shape class. The percentage (yaxis) of SP and BP classes is calculated with translatome-enriched TCs with minimal fold change on $x$-axis. The dashed grep line and yellow line stand for the percentage of SP and BP classes for translatome-depleted TCs with fold change $<0.5$. e The consensus sequence of the immediate downstream $100 \mathrm{nt}$ of the representative TSSs of the top-enriched 200 TCs, top-depleted 200 TCs and randomly picked 200 TCs. The three groups of 100-nt sequences were all analyzed with WebLogo. The $x$-axis shows the relative positions with respect to the representative TSS (at position 1)

than the top-depleted (78) and the randomly picked (97) groups ( $p$-value $<0.001$ by proportion test), showing that the polysome preferentially binds to transcripts with shorter 5' UTR. Sequence motif analysis with MEME identified a significant motif AA(G/A)(A/C)A(G/A)GG in the downstream $100 \mathrm{nt}$ sequences for the topenriched 200 TCs ( $p$-value<0.001), which was not present in the other two groups [32]. Further analysis using Tomtom showed that this motif was not similar to any protein-binding motif ( $p$-value $>0.05$ ), indicating it could be a novel motif [33]. Importantly, compared with the other two groups, there was a significant enrichment ( $>6$ fold) of TATA box in the upstream $100 \mathrm{nt}$ of the top-enriched $200 \mathrm{TCs}$, suggesting this cis-regulatory element played important roles beyond transcription regulation.

\section{Selective usage of genes by Polysome}

Suppose a gene $A$ could generate transcripts from $n$ different core promoters ( $n$ is determined by GRCh37 annotations from Ensembl), we used $\left\{p_{1}, p_{2}, \ldots, p_{n}\right\}$ and $\left\{t_{1}, t_{2}\right.$, $\left.\ldots, t_{n}\right\}$ to denote the abundance of core promoter-derived $\mathrm{TCs}$ in translatome and transcriptome, respectively. We first defined gene $A$ 's abundance in translatome $\left(E_{p}\right)$ and transcriptome $\left(E_{t}\right)$ as follows:

$$
E_{p}=\Sigma_{i=1}^{n} p_{i} ; E_{t}=\sum_{i=1}^{n} t_{i}
$$

We then defined a fold-change score $\left(S_{f_{c}}\right)$ as follows to measure polysome preference toward genes:

$$
S_{f c}=\frac{E_{p}}{E_{t}}=\frac{\sum_{i=1}^{n} p_{i}}{\sum_{i=1}^{n} t_{i}}
$$

We calculated $S_{f c}$ for each gene and the corresponding $p$-value with the DEGseq package (Additional file 8: Table S5). In total, we identified 5804 (49.5\%) genes with significantly changed abundance (i.e., $\left|\log _{2} S_{f c}\right| \geq 1$ and $p$-value $<$ $0.05)$. We then looked into the top 50 translatome-enriched genes (all $S_{f_{c}}>2$ ) ranked by the $p$-values (Table 1) and found that the translatome-enriched ones were highly enriched in the gene families of histones and ribosomal proteins (both $\mathrm{p}$-values $<0.001$ by fisher's exact test). By contrast, the top 50 translatome-depleted genes (all $S_{f_{c}}<$
0.5) enriched in the RNA binding motif containing genes (p-value $<0.001$ ), included no histone or ribosomal genes. Considering that histones and ribosomal proteins are highly abundant in cells and polysome association is a prerequisite for translation [10,34], we infer that polysome selection is an important contributing factor to the abundance. To support this point, we picked three groups of genes (translatome-enriched, translatome-depleted and unchanged) with similar average RPM in transcriptome (see Methods). We found that the protein abundance of translatome-enriched genes was significantly higher than the other two groups (p-value $<0.001$ by Wilcoxon test), while enrichment-unchanged group had much higher abundance than that of translatome-depleted genes ( $\mathrm{p}$-value $<0.001$ ), thus substantiating our inference (see Methods).

As differential usage of core promoters from the same gene could have profound impact on the protein products and the related biological functions [19, 21], we formulated another score $\left(S_{d u}\right)$ as follows to measure the degree of differential usage:

$$
S_{d u}=\frac{1}{2} \quad \sum_{i=1}^{n}\left|\frac{p_{i}}{E_{p}}-\frac{t_{i}}{E_{t}}\right|
$$

This equation applies only when gene A's abundance is $>0$ in both translatome and transcriptome (i.e., $E_{p}>0$ and $\left.E_{t}>0\right)$. Based on this equation, we could easily conclude that: (1) $S_{d u} \in[0,1]$; (2) $S_{d u}=0$ when gene $A$ only uses 1 core promoter (i.e., $n=1$ ), or uses $\geq 2$ core promoters (i.e., $n \geq 2$ ) but the core promoter-derived TCs account for the same proportions between translatome and transcriptome (i.e., $\frac{p_{i}}{E_{p}}=\frac{t_{i}}{E_{t}}$ given that $2 \leq i \leq n$ ); (3) $S_{d u}=1$ when gene A only uses TCs (in translatome) that are not detected in transcriptome by the Illumina sequencing. For simplicity, we only calculated $S_{d u}$ for genes using at least 2 core promoters (i.e., $n \geq 2$ ) in HEK293 cell line. Importantly, we found that, the correlation between $S_{d u}$ and $S_{f c}$ is very low (Pearson correlation $R=-0.04$ ), demonstrating that $S_{d u}$ give additional information independent of $S_{f c}$. We identified 62 genes with $S_{d u}>0.5$ and further analysis of them showed that they were enriched in the myocardin gene family ( $p$-value $<0.001$ by Fisher's exact test). By contrast, the 
Table 1 The top 50 genes that are most enriched or depleted in translatome. Genes are ranked by $p$-values (all $p$-values $<0.001$ ). The histone genes are marked in green, the ribosomal protein genes are in red and the RNA binding motif containing genes in blue. "\#" stands for "number"

top 62 genes with smallest $S_{d u}$ were only enriched in the gene families of histones and ribosomal proteins (p-value $<0.001$ ), suggesting that differential usage of core promoters was very rare for histone and ribosomal genes in HEK293 cell line. Here we listed $>4700$ genes (including the aforementioned $62 \times 2$ genes) with their $S_{d u}$ scores in Additional file 9: Table S6 to spur interest of biologists for the underlining mechanism leading to this differential usage.

\section{Conclusions}

In this work, we use CAGE followed by deep sequencing to systematically compare the transcript $5^{\prime}$ ends between the translatome and transcriptome of human HEK293 cells. The revealed preferential usage of many $5^{\prime}$ ends by polysome shows that, after transcriptional selection of TSS and core promoters, the translation machinery again makes such selection. This comparison leads to the identification of highly selected TSSs, core promoters and gene products in translatome. It also gives rise to the transcription initiation patterns and the sequence characteristics highly correlated with polysome selection. These findings delineate an indirect selection toward TSSs and core promoters by the translation machinery, emphasizing closer than expected interplay between transcription and translation.

\section{Methods}

Growth conditions and RNA isolation

HEK293 cells were cultured in Dulbecco's Minimal Essential Medium (GIBCO, Life Technologies, Carlsbad, CA, USA) supplemented with $10 \%$ FBS (GIBCO \#10099-141), 100 units/ml penicillin, $100 \mu \mathrm{g} / \mathrm{ml}$ streptomycin (GIBCO \#15140-122) and $2 \mathrm{mML}$-glutamine (Sigma) at $37{ }^{\circ} \mathrm{C}$ and $5 \% \mathrm{CO}_{2}$.

Polysome fraction is isolated by $10-50 \%$ sucrose gradient using the method from Bor et al. (2006) with minor modifications [35]. In brief, around $80 \%$ confluent cells were incubated with $50 \mu \mathrm{g} / \mathrm{ml}$ cycloheximide for $30 \mathrm{~min}$ at $37^{\circ} \mathrm{C}$. Cells were scrapped into a $1.5 \mathrm{ml}$ Eppendoff tube with a cell lifter. And cells were lysed by 250ul 2XRSB/RNasin and $250 \mu \mathrm{l}$ of polysome extraction buffer. The polysome fraction was collected with the BioComp piston gradient fractionator after ultra-speed centrifugation with SW41Ti rotor buckets at $36,000 \mathrm{rpm}$ for $2 \mathrm{~h}$.

The cells without cycloheximide treating were lysed with TRIzol reagent (Invitrogen, Cat. No. 15596-018) and total RNA was extracted following TRIzol protocol. 
The polysome-associated RNA was also extracted using TRIzol with the same method.

\section{CAGE library preparation}

Two 27 bp-tagged deep sequencing libraries were prepared using the methods described in Valen et al. (2009) and Takahashi et al. (2012) [17, 36]. In brief, using SuperScript II (Invitrogen), first-strand cDNA was synthesized with $30 \mu \mathrm{g}$ total RNA and $8 \mu \mathrm{g}$ of anchored-N15 primer(5'AAGGTCTATCAGCAGN15). The capped RNA was selected using the cap-trapper method described in Valen et al. (2009) [17]. The 2nd strand DNA was synthesized by ligating a N6 adaptor (CCACCGACAGGTTCAGAGT TCTACAGCTTCAGCAGNNNNNN Phos / N6-down: Phos CTGCTGAAGCTGTAGAACTCTGAACCTGTCG GTGG NH2) to the 3'end of the ssDNA with DNA ligation kit (Takara, Tokyo, Japan). The dsDNA was digested with EcoP15I (NEB, Ipswich, MA, USA) following the method from Takahashi et al. (2012) [36]. A 3' adaptor (3' adaptorup, NNTCGTATGCCGTCTTCTGCTTG / 3' adaptordown: CAAGCAGAAGACGGCATACGA) was ligated to the recovered EcoP15I fragments. The PCR primer1 (5'CAAGCAGAAGACGGCATACGA -3') and PCR primer2 (5'-AATGATACGGCGACCACCGACAGGTTCAG

AGTTCTACAGTCCGA -3') were used to create deep sequencing libraries which contained the first $27 \mathrm{bp}$ from the $5^{\prime}$ ends of capped RNA. The two libraries were prepared and sent out for sequencing together. Sequencing was performed using Solexa GAII following the manufacturer's protocol. Sequencing data from different batches (to achieve enough sequencing depth) were merged together before downstream data analysis.

\section{Quality control and sequencing reads alignment}

Raw sequencing data were first processed using fqtrim (version 0.94) to remove the $3^{\prime}$ sequencing adaptor, 5' barcodes (CTTCAGCAG and GATCAGCAG for translatome and transcriptome RNA library respectively) and low-quality reads (with parameters -q $20-\mathrm{m} \mathrm{1}$ ) [23, 27]. Reads with length $\geq 30$ or $\leq 24$ were also removed from downstream analysis since the expected length of CAGE tags was $27 \mathrm{nt}$ based on the protocols above [35, 36]. Bowtie was then used to map the clean reads to the human genome (assembly GRCh37) with two mismatches allowed (using parameters -v 2 --best --strata - m 1). Only uniquely mapped reads were used for further analysis. The R package - CAGEr was used to correct "G" nucleotide addition bias at the 5 ' ends of CAGE tags introduced in the library preparation [28].

\section{Tag clustering and TC distribution}

Tag clusters were identified with the following two steps. First, tags that overlapped on the same strand were grouped into a tag set. Second, any tag set with at least four tags were defined as a tag cluster. Suppose tags randomly distributed on the human genome were background noise, the probability $n$ tags were observed in a tag set of $(n-1) \times 27$ nt length follows a Poisson distribution with $\lambda=\frac{(n-1) \times 27}{N} \times T$ ( $N$ is the human genome length and $T$ is the number of uniquely mapped reads). Based on this $\lambda$, it is obvious that the probability of $n \geq 4$ is less than 0.001 , which corresponds to the $p$-value. Therefore, the above two steps guaranteed that the identified tag clusters had significant $p$-values and were not background noise.

Different genomic features could overlap and the positions of TCs could be situated within two or more features at the same time. When this happened, we used the method described in Ref. [3] to assign TCs with the following priority: 5' UTR $>3$ ' UTR $>$ CDS $>$ intron (the $100 \mathrm{nt}$ upstream of $5^{\prime}$ UTR were also included in $5^{\prime}$ UTR) [3]. TCs not mapped to any of the four features in protein-coding genes were considered "intergenic".

\section{Classification criteria for TC shape class}

We classified TCs (with $\geq 100$ tags) into four shape classes with a method similar to those from previous studies $[3,16]$. Briefly, we used the following criteria: $(i)$ a TC was classified into SP class if the distance between the 25th and 75th percentile of its tag positions was $<4 \mathrm{nt}$, or the distance between the 15th and 85th percentile was $<6 \mathrm{nt}$; (ii) if a TC did not meet (i) but the ratio between its highest peak and second highest peak was $>2$ and the highest peak accounted for $>20 \%$ of all tags in it, the TC was classified into DP class; (iii) If distance between any two consecutive peaks (both peaks accounted for $>15 \%$ of all tags) was $>5 \mathrm{nt}$ and the TC was in neither SP nor DP class, it was classified into MP class; (iv) if a TC did not meet (i), (ii) or (iii), the TC was classified into BP class.

\section{Additional definitions, tools and data sources}

In this study, the expressed transcripts were defined as those with at least one TC identified within the $5^{\prime}$ UTR or the $100 \mathrm{nt}$ upstream region. The expressed genes were defined as those with at least one annotated transcript expressed in transcriptome. To make comparison between samples, RPM was used to normalize the read number of each cluster, which was defined as follows:

$$
\begin{aligned}
\mathrm{RPM}= & \frac{\text { the number of reads in a read cluster }}{\text { the total number of mapped reads }} \\
& \times 1000000
\end{aligned}
$$

Statistical analysis (including the hypothesis testing) was performed with the $\mathrm{R}$ language (http://www.r-project.org/). In the case of multiple hypothesis testing, we used $\mathrm{BH}$ method to correct $p$-values (unless otherwise 
specified) [37]. Sequence and motif analysis was performed based on R, WebLogo (http://weblogo.berkeley. edu) and MEME (http://meme-suite.org/tools/meme, with the option "search given stand only" for motif search only in the RNA transcripts) and Tomtom [http://meme-suite.org/tools/tomtom, with Vertebrates (In vivo and in silico) as the database of known motifs] [31-33]. Multiple R packages and tools were used in DNA sequence retrieval and figure preparation [38-43].

All CAGE data from the FANTOM5 project were downloaded from the FANTOM website (http://fantom. gsc.riken.jp/5/datafiles/latest/), including the TCs and the representative TSSs for 180 human tissues and primary cells (see Additional file 9: Table S6 for more details of the 180 samples) [29]. The category of gene families and their members were retrieved from HGNC website (https://www.genenames.org/) [44].

\section{Polysome selection and protein abundance}

We picked three groups of genes with the following criteria: (1) picked top 100 genes from the translatomeenriched genes (ranked by p-values; all $S_{f_{c}}>2$ ); (2) picked top 100 genes from the unchanged genes (i.e., $0.9<S_{f_{c}}<$ 1.1; genes were ranked by their RPM in transcriptome); (3) picked the genes ranked from 101th to 200th from the translatome-depleted genes (ranked by p-values; all $S_{f_{c}}<$ 0.5 ). This way, we obtained three groups consisting of 100 genes each with similar average RPM in transcriptome (290.3, 298.8, 300.4 for translatome-enriched, unchanged, translatome-depleted genes, respectively). We retrieved protein abundance data (i.e., average protein copy number) in mouse fibroblasts (NIH3T3 cell line) from Ref. [34] Additional file 5: Table S3. We identified the homologous proteins between mouse and human based on HGNC nomenclature and used mouse proteins' abundance to represent the homologs' abundance in human [44].

\section{Accession number}

Raw sequencing data used in this work are available in the ArrayExpress database (http://www.ebi.ac.uk/arrayexpress) under accession number E-MTAB-7382.

\section{Additional files}

Additional file 1: Figure S1. Tag distribution around annotated TSSs of human transcripts. The black line stands for translatome and the red line stands for transcriptome. The TSS annotation was retrieved from human GRCh37 annotations downloaded from Ensembl. (PNG $188 \mathrm{~kb}$ )

Additional file 2: Figure S2. Typical examples for the 4 TC shape classes. SP class (A) are characterized by a sharp peak that stands for the majority of tags in a TC. BP class (D) do not have any peak much stronger than the others in a TC. The $5^{\prime}$ end distributions in DP (B) and MP (C) classes are somewhere between SP and BP classes (refer to
Methods for more details). The TC information (chromosome, strand and genomic position) are placed on top of each example. (PNG $336 \mathrm{~kb}$ )

Additional file 3: Table S1. TCs with changed 5 ' end distributions between translatome and transcriptome. "Chr" stands for "chromosome". "TC start" and "TC end" gives the genomic range of TCs on the human genome. "Gene Symbol" shows the genes where TCs are located. "Source" shows where TCs come from. P-values are calculated with KS test and adjusted with BH method. Any two TCs in sequential rows from Translatome and transcriptome correspond to the same core promoter and thus have the same $p$-value. (XLSX $268 \mathrm{~kb}$ )

Additional file 4: Table S2. Length difference of the $5^{\prime}$ UTR between translatome and transcriptome. "Length Difference" shows the difference in length of the 5' UTR. "Length Status" shows whether the length of the $5^{\prime} \mathrm{UTR}$ in translatome is the same as, or shorter than, or longer than that in transcriptome. The definitions of the other column names are the same as in Additional file 3: Table S1. (XLSX $1009 \mathrm{~kb}$ )

Additional file 5: Table S3. Usage frequency of HEK293-derived representative TSSs in 180 human samples. All 3248 representative TSSs are from HEK293 translatome. In the table, "1" means the representative TSS (row name) is also used as representative TSS in the sample (column name). The name of each representative TSS contains the information of chromosome, strand and genomic position. (XLSX $1773 \mathrm{~kb}$ )

Additional file 6: Table S4. TCs with significantly changed RPM between translatome and transcriptome. "z-score", "p-value" and "q-value" are calculated with the R package of DEGseq. TCs are ranked by the qvalues. (XLSX $2126 \mathrm{~kb}$ )

Additional file 7: Calculation of TC fold change (FC) with polysome-free RNA instead of total RNA. (DOCX $13 \mathrm{~kb}$ )

Additional file 8: Table S5. Comparison of genes' abundance between translatome and transcriptome. " $E_{p}$, " $E_{t}$ " and "S $S_{c}$ " are all defined in the main text. "p-value" and "q-value" are calculated with the R package of DEGseq. Genes are ranked by the q-value. Genes with too few reads to calculated the $p$-values are removed from this table. (XLSX $925 \mathrm{~kb}$ )

Additional file 9: Table S6. Differential usage of core promoters from the same gene by polysome. Under each gene name, there are two (or more) rows corresponding to two (or more) core promoters of the gene. Each gene has one $S_{d u}$ score, which is defined in the main text. (XLSX $660 \mathrm{~kb})$

\section{Abbreviations}

CAGE: Cap Analysis of Gene Expression; CDS: Coding sequence; FC: Fold change; KS test: Kolmogorov-Smirnov test; RPM: Reads per million; TC: Tag cluster; TSS: Transcription start site; UTR: Untranslated region

\section{Acknowledgements}

We thank Dr. Xiaodong Zhao, Dr. Daniel M. Czajkowsky and Dr. Pan Tong for their thoughtful discussion. We also thank Mr. Ziwen Guo, Ms. Yuan Wang and Ms. Hualei Kong for their help in figure preparation.

\section{Funding}

This study was supported by National Natural Science Foundation of China (31501054, 11374207, 81627801), MOST (No. 2014YQ090709), the Open Large Infrastructure Research of Chinese Academy of Sciences (18H100000104), the Science and Technology Commission of Shanghai Municipality (No.

17JC1400804), Laboratory Innovative Research Program of Shanghai Jiao Tong University (175J-18). The funding bodies had no roles in the design of the study and collection, analysis, and interpretation of data and in writing the manuscript.

\section{Availability of data and materials}

All data generated and analyzed in this study were publicly available (see Methods)

\section{Authors' contributions}

ZS conceived the study and designed the experiments; $\mathrm{HL}$ and NZ performed the data analysis; $L B$ and $H L$ carried out the experiments; $X L$, YK and JS contributed reagents; $\mathrm{HL}$ and BL wrote the manuscript. All authors have read, revised and approved the final manuscript. 


\section{Ethics approval and consent to participate}

Not applicable.

\section{Consent for publication}

Not applicable.

\section{Competing interests}

The authors declare that they have no competing interests.

\section{Publisher's Note}

Springer Nature remains neutral with regard to jurisdictional claims in published maps and institutional affiliations.

\section{Author details}

${ }^{1}$ State Key laboratory for Oncogenes and Bio-ID Center, School of Biomedical Engineering, Shanghai Jiao Tong University, 800 Dongchuan Road, Shanghai 200240, China. ${ }^{2}$ Instrumental Analysis Center, Shanghai Jiao Tong University, 800 Dongchuan Road, Shanghai 200240, China. ${ }^{3}$ Key Laboratory of Systems Biomedicine (Ministry of Education), Shanghai Center for Systems Biomedicine, Shanghai Jiao Tong University, 800 Dongchuan Road, Shanghai 200240, China.

\section{Received: 25 November 2018 Accepted: 27 March 2019}

\section{Published online: 11 April 2019}

\section{References}

1. Lenhard B, Sandelin A, Carninci P. Metazoan promoters: emerging characteristics and insights into transcriptional regulation. Nat Rev Genet. 2012;13(4):233-45.

2. Hoskins RA, Landolin JM, Brown JB, Sandler JE, Takahashi H, Lassmann T, et al. Genome-wide analysis of promoter architecture in Drosophila melanogaster. Genome Res. 2011;21(2):182-92.

3. Li H, Hou J, Bai L, Hu C, Tong P, Kang Y, et al. Genome-wide analysis of core promoter structures in Schizosaccharomyces pombe with DeepCAGE. RNA Biol. 2015;12(5):525-37.

4. Arribere JA, Gilbert W. Roles for transcript leaders in translation and mRNA decay revealed by transcript leader sequencing. Genome Res. 2013;23(6): 977-87

5. Ingolia NT, Ghaemmaghami S, Newman JRS, Weissman JS. Genome-wide analysis in vivo of translation with nucleotide resolution using ribosome profiling. Science. 2009;324(5924):218-23.

6. Halbeisen RE, Galgano A, Scherrer T, Gerber AP. Post-transcriptional gene regulation: from genome-wide studies to principles. Cell Mol Life Sci. 2008; 65(5):798-813.

7. Keene JD. RNA regulons: coordination of post-transcriptional events. Nat Rev Genet. 2007:8(7):533-43.

8. Bilanges B, Stokoe D. Mechanisms of translational deregulation in human tumors and therapeutic intervention strategies. Oncogene. 2007;26(41):5973-90.

9. Halbeisen RE, Gerber AP. Stress-dependent coordination of transcriptome and translatome in yeast. PLoS Biol. 2009:7(5):e1000105.

10. Jackson RJ, Hellen CU, Pestova TV. The mechanism of eukaryotic translation initiation and principles of its regulation. Nat Rev Mol Cell Biol. 2010;11(2): $113-27$.

11. Wang X, Hou J, Quedenau C, Chen W. Pervasive isoform-specific translational regulation via alternative transcription start sites in mammals. Mol Syst Biol. 2016;12(7):875.

12. Hou J, Wang X, McShane E, Zauber H, Sun W, Selbach M, et al. Extensive allelespecific translational regulation in hybrid mice. Mol Syst Biol. 2015;11(8):825.

13. Thoreen CC, Chantranupong L, Keys HR, Wang T, Gray NS, Sabatini DM. A unifying model for mTORC1-mediated regulation of MRNA translation. Nature. 2012:485(7396):109-13.

14. Calvo SE, Pagliarini DJ, Mootha VK. Upstream open reading frames cause widespread reduction of protein expression and are polymorphic among humans. Proc Natl Acad Sci U S A. 2009;106(18):7507-12.

15. Shiraki T, Kondo S, Katayama S, Waki K, Kasukawa T, Kawaji H, et al. Cap analysis gene expression for high-throughput analysis of transcriptional starting point and identification of promoter usage. Proc Natl Acad Sci U S A. 2003;100(26):15776-81

16. Carninci P, Sandelin A, Lenhard B, Katayama S, Shimokawa K, Ponjavic J, et al. Genome-wide analysis of mammalian promoter architecture and evolution. Nat Genet. 2006;38(6):626-35.
17. Valen E, Pascarella G, Chalk A, Maeda N, Kojima M, Kawazu C, et al. Genome-wide detection and analysis of hippocampus core promoters using DeepCAGE. Genome Res. 2009;19(2):255-65.

18. Brown JB, Boley N, Eisman R, May GE, Stoiber MH, Duff MO, et al. Diversity and dynamics of the Drosophila transcriptome. Nature. 2014,512(7515):393-9.

19. Ushijima T, Hanada K, Gotoh E, Yamori W, Kodama Y, Tanaka H, et al. Light controls protein localization through Phytochrome-mediated alternative promoter selection. Cell. 2017;171(6):1316-25.

20. Tamarkin-Ben-Harush A, Vasseur JJ, Debart F, Ulitsky I, Dikstein R. Capproximal nucleotides via differential elF4E binding and alternative promoter usage mediate translational response to energy stress. Elife. 2017;6:e21907.

21. Cheng Z, Otto GM, Powers EN, Keskin A, Mertins P, Carr SA, et al. Pervasive, coordinated protein-level changes driven by transcript isoform switching during meiosis. Cell. 2018;172(5):910-23.

22. Kratz A, Beguin P, Kaneko M, Chimura T, Suzuki AM, Matsunaga A, et al. Digital expression profiling of the compartmentalized translatome of Purkinje neurons. Genome Res. 2014;24(8):1396-410.

23. Pertea G. gpertea/fqtrim:fatrim release v0.9.7; 2018.

24. Langmead B, Trapnell C, Pop M, Salzberg SL. Ultrafast and memory-efficient alignment of short DNA sequences to the human genome. Genome Biol. 2009;10(3):R25.

25. Consortium F, the RP, Clst, Forrest AR, Kawaji H, Rehli M, et al. A promoterlevel mammalian expression atlas. Nature. 2014;507(7493):462-70.

26. Zhang X, Gao X, Coots RA, Conn CS, Liu B, Qian SB. Translational control of the cytosolic stress response by mitochondrial ribosomal protein L18. Nat Struct Mol Biol. 2015;22(5):404-10.

27. Wiesner T, Lee W, Obenauf AC, Ran L, Murali R, Zhang QF, et al. Alternative transcription initiation leads to expression of a novel ALK isoform in cancer. Nature. 2015;526(7573):453-7.

28. Haberle V, Forrest AR, Hayashizaki Y, Carninci P, Lenhard B. CAGEr: precise TSS data retrieval and high-resolution promoterome mining for integrative analyses. Nucleic Acids Res. 2015;43(8):e51

29. Lizio M, Harshbarger J, Shimoji H, Severin J, Kasukawa T, Sahin S, et al. Gateways to the FANTOM5 promoter level mammalian expression atlas. Genome Biol. 2015;16(1):22.

30. Wang L, Feng Z, Wang X, Wang X, Zhang X. DEGseq: an $R$ package for identifying differentially expressed genes from RNA-seq data. Bioinformatics. 2009:26(1):136-8.

31. Crooks GE, Hon G, Chandonia JM, Brenner SE. WebLogo: a sequence logo generator. Genome Res. 2004;14(6):1188-90.

32. Bailey TL, Boden M, Buske FA, Frith M, Grant CE, Clementi L, et al. MEME SUITE: tools for motif discovery and searching. Nucleic Acids Res. 2009; 37(Web Server):W202-8.

33. Gupta S, Stamatoyannopoulos JA, Bailey TL, Noble WS. Quantifying similarity between motifs. Genome Biol. 2007:8(2):R24.

34. Schwanhausser B, Busse D, Li N, Dittmar G, Schuchhardt J, Wolf J, et al. Global quantification of mammalian gene expression control. Nature. 2011; 473(7347):337-42

35. Bor YC, Swartz J, Li Y, Coyle J, Rekosh D, et al. Northern blot analysis of mRNA from mammalian polyribosomes. Nat Protoc. 2006.

36. Takahashi H, Lassmann T, Murata M, Carninci P. 5 ' end-centered expression profiling using cap-analysis gene expression and next-generation sequencing. Nat Protoc. 2012;7(3):542-61.

37. Benjamini $Y$, Hochberg $Y$. Controlling the false discovery rate: a practical and powerful approach to multiple testing. J R Stat Soc Ser B Methodol. 1995:289-300.

38. Pagès $H$, Aboyoun $P$, Gentleman R, DebRoy S. Biostrings: Efficient manipulation of biological strings. 2019. R Package Version 2.50.2.

39. Li H, Su X, Gallegos J, Lu Y, Ji Y, Molldrem JJ, et al. dsPIG: a tool to predict imprinted genes from the deep sequencing of whole transcriptomes. BMC Bioinformatics. 2012;13(1):271.

40. Wickham H. ggplot2: Elegant Graphics for Data Analysis. New York: Springer-Verlag; 2009. ISBN 978-3-319-24277-4

41. Kampstra P. Beanplot: a boxplot alternative for visual comparison of distributions. J Stat Softw. 2008:28:1-9.

42. Peng G, Wilson $R$, Tang $Y$, Lam T, Nairn AC, Williams $K$, et al. ProteomicsBrowser: MS/proteomics data visualization and investigation. Bioinformatics, bty958, https://doi.org/10.1093/bioinformatics/bty958.

43. Cai G, Liang S, Zheng X, Xiao F. Local sequence and sequencing depth dependent accuracy of RNA-seq reads. BMC Bioinformatics. 2017;18(1):364.

44. Yates B, Braschi B, Gray KA, Seal RL, Tweedie S, Bruford EA. Genenames. org: the HGNC and VGNC resources in 2017. Nucleic Acids Res. 2016:gkw1033. 